\title{
Prevalência de retinopatia diabética em pacientes atendidos pela Estratégia Saúde da Família no município de Ananindeua - PA
}

\author{
Prevalence of diabetic retinopathy in patients assisted by a unit of Family Health Strategy in the city of \\ Ananindeua, Brazil
}

\section{Prevalencia de la retinopatía diabética en pacientes atendidos por la Estratégia de Salud Familiar en el município de Ananindeua - PA}

Dyndara Rodrigues Pedrosa. Universidade Federal do Pará (UFPA). dynda_@hotmail.com (Autora correspondente)

Emanuelle Oliveira Lemos. Universidade Federal do Pará (UFPA).emanuelle.med.ufpa@hotmail.com

Débora Cristina de Abreu Gonçalves. Universidade Federal do Pará (UFPA). deboracnut@hotmail.com

Perla Suely Gaia Raniéri. Universidade Federal do Pará (UFPA). perlaranieri@yahoo.com.br

Carla Andréa Avelar Pires. Universidade Federal do Pará (UFPA). carlaavelarpires@bol.com.br

Valter Resende de Paiva. Universidade Federal do Pará (UFPA). valter_paiva@hotmail.com

\section{Resumo}

Objetivo: Identificar a prevalência de retinopatia diabética (RD) em pacientes atendidos pela Estratégia Saúde da Família (ESF) de Ananindeua, Pará. Métodos: Este é um estudo do tipo seccional com 40 pacientes portadores de Diabetes Mellitus (DM) cadastrados no programa HIPERDIA e assistidos pela ESF de Ananindeua. Os selecionados foram submetidos a um questionário padronizado domiciliar e responderam questões quanto ao conhecimento, evolução e complicações do DM e da RD, além de dados socioeconômicos. Na etapa seguinte, um médico oftalmologista voluntário realizou fundoscopia e detectou os portadores da RD que, em seguida, foram encaminhados para um centro especializado para receber 0 tratamento espećifico. Desses pacientes, 9 foram excluídos da pesquisa por não comparecerem na unidade para realizar a fundoscopia. Resultados: A prevalência de RD encontrada foi de 40,7\%, sendo que 4 pacientes tiveram sua análise prejudicada devido à presença de catarata, portanto só foi possível fazer avaliação em 27. Fatores associados com RD, após análise das variáveis, foram: tempo de conhecimento do diagnóstico de ser portador de DM, tipo de DM e RD não proliferativa. Conclusão: Este estudo piloto ressalta a importância da atenção primária no acompanhamento dos pacientes diabéticos, e da orientação aos servidores da saúde pública quanto à necessidade do encaminhamento periódico dos diabéticos ao oftalmologista.

\section{Abstract}

Objective: To identify the prevalence of diabetic retinopathy (DR) in patients served by the Family Health Strategy (FHS) of Ananindeua, Brazil. Methods: This was a crosssectional study with 40 patients with diabetes mellitus (DM) enrolled in the HIPERDIA program and assisted by the FHS of Ananindeua. Those selected were subjected to a standardized questionnaire at home and answered questions about the knowledge, outcomes and complications of DM and DR, and socioeconomic data. In the next step, a volunteer ophthalmologist performed ophthalmoscopy and found those with DR, who were sent to a specialized center to receive treatment. Of these patients, 9 were excluded because they did not attend the unit to perform ophthalmoscopy. Results: The prevalence of DR was found to be 40.7\%; however, 4 patients had impaired their analysis due to the presence of cataracts, so it was only possible to do evaluation in 27 . Factors associated with DR, after analysis of the variables were the time to the patients be aware of the diagnosis of DM, type of DM and non-proliferative DR. Conclusion: This pilot study underscores the importance of primary care in monitoring diabetic patients and guidance to the professionals of public health about the need for periodic referral of diabetic patients to ophthalmologists.

\section{Resumen}

Objetivo: Identificar la prevalencia de la retinopatía diabética (RD) en pacientes atendidos por la Estrategia de Salud Familiar del Ananindeua, Pará. Métodos: Este es un estudio de la sección con 40 pacientes con diabetes mellitus (DM) inscritos en el programa HIPERDIA y acompañado por ESF del Ananindeua. Los seleccionados fueron sometidos a un cuestionario estandarizado en sus casas y respondieron a preguntas sobre el conocimiento, la evolución y las complicaciones de la RD y los datos socioeconómicos. En el siguiente paso, un oftalmólogo voluntario realizó fondo de ojo y encontró que aquellos con RD, y luego fueron derivados a un centro especializado para recibir tratamiento. De estos pacientes, 9 fueron excluidos del estudio por no asistir a la unidad para realizar fondo de ojo. Resultados: La prevalencia de RD se encontró que era del 40,7\%, y 4 pacientes tenían afectada su análisis debido a la presencia de cataratas, por lo que sólo era posible para evaluar 27. Los factores asociados con RD, tras el análisis de las variables, eran: tiempo para estar al tanto del diagnóstico de diabetes, tipo de diabetes y la retinopatía diabética no proliferativa. Conclusión: Este estudio piloto subraya la importancia de la atención primaria en el seguimiento de los pacientes diabéticos, la salud y orientación a los funcionarios sobre la necesidad de derivación periódica de los diabéticos a un oftalmólogo.

Como citar: Pedrosa DR, Lemos EO, Gonçalves DCA, Raniéri PSG, Pires CAA, Paiva VR. Prevalência de retinopatia diabética em pacientes atendidos pela Estratégia Saúde da Família no município de Ananindeua - PA. Rev Bras Med Fam Comunidade. 2013;8(26):58-63. Disponível em: http://dx.doi.org/10.5712/rbmfc7(23)394
Palavras-chave: Retinopatia Diabética Diabetes Mellitus Estratégia Saúde da Família

Keywords:

Diabetic Retinopathy Diabetes Mellitus Family Health Strategy

Palabras clave: Retinopatía Diabética Diabetes Mellitus Estrategia de Salud Familia 


\section{Introdução}

O Diabetes Mellitus (DM) é uma das principais doenças crônicas que acomete populaçôes em todo o mundo, de diferentes níveis sociais e econômicos. A prevenção, o diagnóstico e o tratamento vêm se tornando alvos da Saúde Pública, devido ao crescente aparecimento de novos casos. A incidência da DM tipo 2 aumentou consideravelmente no mundo atual devido: ao maior consumo de dieta hipercalórica; ao aumento da expectativa de vida e da taxa de urbanizaçáo; e a mudanças do estilo de vida. Além disso, esses fatores são associados às alteraçôes genéticas a que todos os pacientes estão susceptíveis ${ }^{1}$.

Algumas patologias se associam ao diabetes, como, por exemplo, a retinopatia, a neuropatia e a nefropatia. A Retinopatia Diabética $(\mathrm{RD})$ é a principal causa de cegueira entre os diabéticos ${ }^{2-4}$. Esta complicação ocorre devido à grande concentração de glicose na circulação sanguínea, o que lesa os vasos sanguíneos que nutrem a retina. A diminuição da disponibilidade de vasos fisiologicamente normais que possam nutrir e oxigenar a retina provoca hipóxia tecidual, o que resulta em uma diminuição da acuidade visual ao longo dos estágios dessa patologia 5 .

Atualmente são escassos os estudos referentes à prevalência de RD no Brasil. Estudos feitos em distintas regiôes do país demonstram prevalência da $\mathrm{RD}$ variando de $24 \%$ a 39,4\%, sendo mais frequente em pacientes que residem em regiôes metropolitanas. Através de inferências estatísticas, hoje se estima um número aproximado de 2 milhóes de brasileiros com $\mathrm{RD}$, levando em conta que uma parte desses indivíduos terá alguma perda visual relacionada à doença ${ }^{6}$. Estima-se que 50\% dos pacientes portadores de DM sejam afetados pela $\mathrm{RD}$, sendo responsável por 7,5\% das causas de incapacidade de adultos para a prática do trabalho e por 4,58\% das deficiências visuais 5 .

Apesar da grande prevalência e incidência da $\mathrm{RD}$, a quantidade de centros que proporcionam os equipamentos e profissionais qualificados para o diagnóstico pode ser considerada ainda insuficiente. No Brasil, estima-se que existam 48.707 ambulatoriais oftalmológicos. Destes, $3.419(7 \%)$ atendem a população que utiliza o SUS e somente $52(1,52 \%)$ destes ambulatórios do SUS, estáo no Estado do Pará7. A magnitude da DM e suas sequelas, bem como a baixa cobertura de profilaxia para as suas complicaçóes, faz com que seja de suma importância a realização de pesquisas sobre RD que acomete os usuários do SUS . $^{8}$

A Estratégia Saúde da Família (ESF), além de ser uma importante fonte de acesso e cuidado para a população assistida, deve contribuir para detectar de forma precoce as complicaçôes ocasionadas pelo DM, como a RD ${ }^{7}$.

O presente estudo teve como objetivo identificar a prevalência de retinopatia diabética em pacientes atendidos pela Estratégia Saúde da Família Cidade Nova VI no município de Ananindeua-Pa.

\section{Metodologia}

Este é um estudo com delineamento epidemiológico do tipo seccional, observacional, de natureza descritiva. A pesquisa foi realizada de agosto de 2010 a fevereiro de 2011, com uma amostra de conveniência, constituída de 40 pacientes portadores de Diabetes Mellitus cadastrados e tratados no programa HIPERDIA da Estratégia Saúde da Família Cidade Nova VI, localizada no município de Ananindeua-Pará.

No período de agosto a outubro de 2010, ocorreu a primeira etapa do projeto que consistiu nas visitas domiciliares a 70 pacientes diabéticos cadastrados no programa Hiperdia. Foram incluídos no estudo 40 destes 70 pacientes, pois é o total de pacientes com DM acompanhados e tratados na ESF citada, enquanto que os demais foram excluídos da amostra por fazerem tratamento em instituição privada. Esta etapa teve a finalidade de cadastrar e de coletar dados dos pacientes a partir de um questionário padronizado, desenvolvido pelos autores, obtendo resultados quanto ao conhecimento, evolução e complicaçôes do DM e da RD, além de dados socioeconômicos.

No mês de novembro, os pacientes selecionados foram convocados a participar da segunda etapa do projeto. No dia da ação, 31 pacientes compareceram à Unidade Básica de Saúde e nove foram excluídos por não comparecerem. A atividade foi iniciada com orientaçóes sobre a RD, o exame de fundoscopia e os objetivos do estudo. Posteriormente, foi apresentado um vídeo com informaçôes didáticas sobre Diabetes Mellitus e RD, além de fotos registradas ao longo das visitas domiciliares.

$\mathrm{Na}$ etapa seguinte, um médico oftalmologista voluntário, especialista em retina, realizou fundoscopia binolocular nos pacientes diabéticos previamente selecionados e detectou os portadores da RD. Este exame foi realizado inicialmente com a aplicação de um colírio que dilata a pupila. Em seguida, através de um oftalmoscópico direto ou indireto (com auxílio 
de lentes de aumento), um feixe de luz é emitido em direção à pupila com o objetivo de visualizar os vasos sanguíneos presentes no globo ocular. O exame permite a avaliação de alterações da microcirculação, assim sendo, diagnostica a RD 9 . Em seguida, aqueles diagnosticados foram encaminhados para um centro especializado para receber o tratamento específico.

Todos os pacientes que fizeram parte da pesquisa compreenderam e assinaram o Termo de Consentimento Livre e Esclarecido aprovado pelo Comitê de Ética em Pesquisa com Seres Humanos da Universidade Federal do Pará (Protocolo $\left.n^{\circ} 093 / 2010\right)$.

Os resultados obtidos foram organizados em uma planilha de dados do Excel $^{\circledR} 2007$ e receberam tratamento estatístico a partir do programa Epi Info 3.5.2. Inicialmente, foi realizada análise descritiva dos dados e, em seguida, a análise estatística inferencial com os testes do Quiquadrado e exato de Fisher. Estabeleceu-se nível $\alpha$ de significância a valores iguais ou menores que 0,05 .

\section{Resultados}

Em relação ao perfil demográfico encontrado neste estudo, a maioria dos entrevistados era do sexo feminino (64,5\%). A média da idade foi 58,1 anos. Quanto ao grau de escolaridade, a maior frequência foi de pacientes com ensino fundamental incompleto $(58,6 \%)$.

Dos 31 pacientes avaliados pelo exame de fundoscopia, 4 tiveram sua análise prejudicada devido à presença de catarata, portanto só foi possível fazer avaliação em 27 pacientes. Desse grupo, 11 (40,7\%) apresentaram algum grau de RD do tipo náo proliferativa (RDNP), sendo que destes, $6(54,5 \%)$ apresentaram a RDNP leve, 3 (27,3\%) a RDNP incipiente, e 2 $(18,2 \%)$ a RDNP moderada (Figura 1$)$.

Foram avaliados 10 indivíduos do sexo masculino e 17 do sexo feminino. A presença de RD entre os homens foi de $5(50 \%)$ e entre as mulheres foi de $6(35,3 \%)$ (Tabela 1$)$. No entanto, considerando o total de pacientes diagnosticados com $\mathrm{RD}$ (11), não houve diferença significativa na prevalência de RD no sexo feminino (54,5\%) em relação ao sexo masculino $(45,5 \%), \mathrm{p}=0,3632$.

A média de tempo do diagnóstico do DM dos pacientes que apresentaram RD foi de 11,8 anos. Entre o grupo com $\mathrm{RD}$, o item tempo de diagnóstico da doença relatado foi de menos que 8 anos em dois pacientes, entre 8 e 15 anos em sete pacientes e há mais de 15 anos em dois (Tabela 2).

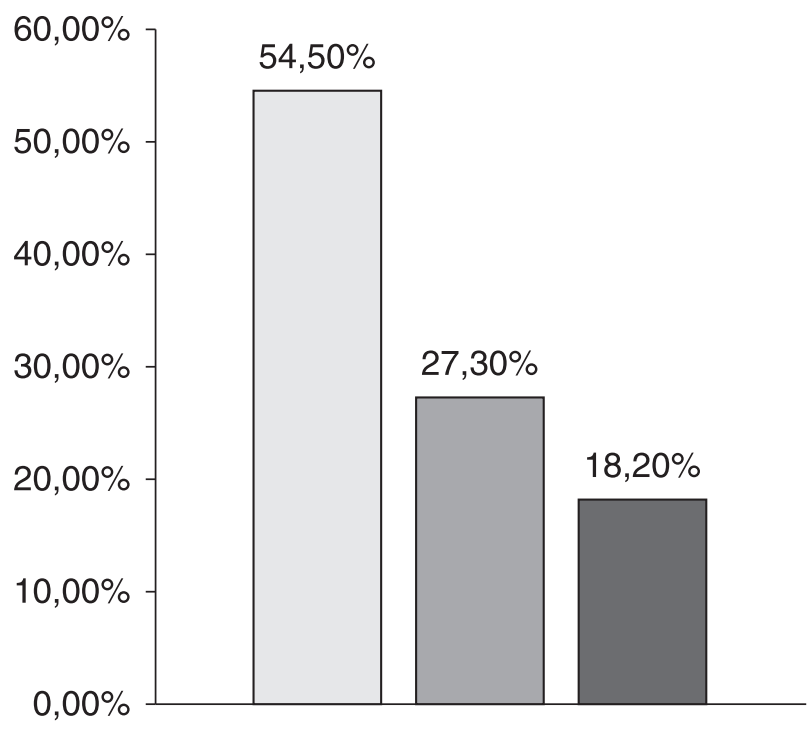

$\square$ R. D. não proliferativa leve

$\square$ R. D. não proliferativa incipiente

- R. D. não proliferativa moderada

Figura 1. Tipos de Retinopatia diagnosticada nos pacientes submetidos ao exame do fundo do olho. 
Em relação ao tratamento para o DM, 58,1\% afirmaram fazer uso de hipoglicemiante oral disponibilizado pela Unidade de Saúde, 22,6\% afirmaram associar medicamentos orais a uma adequada dieta alimentar, 6,5\% recebem insulinizaçáo plena, 3,2\% afirmaram fazer apenas uma adequada dieta alimentar, enquanto que 9,7\% relataram náo fazer qualquer tipo de tratamento (Tabela 3). Náo foi relatado o uso de insulina plena associado a um hipoglicemiante oral e a uma adequada dieta alimentar.

No questionário inicial, todos afirmaram ter conhecimento de que o DM, de alguma maneira, pode comprometer a visão. A maioria dos avaliados (62,5\%) afirmou ser diabético há mais de cinco anos e 41,4\% do total entrevistado acredita que a Unidade de Saúde é o local mais apropriado para tratar os distúrbios visuais decorrentes da doença de base.

Entre os pacientes que consideram e relataram possuir alguma sensação de perda visual, $75 \%$ foram encaminhados pela Unidade de Saúde para um centro especializado em oftalmologia, enquanto que aqueles pacientes que náo consideram possuir alguma sensação de perda visual, 33,3\% foram assim mesmo encaminhados ao oftalmologista por haver suspeita de gravidade na avaliação do médico da unidade de saúde (Figura 2).

Tabela 1. Pacientes segundo sexo e presença de retinopatia diabética.

\begin{tabular}{lcccc}
\multicolumn{1}{r}{ Sexo } & Presença & Ausência & Total & \% de presença \\
\hline Feminino & 6 & 11 & 17 & 35,3 \\
Masculino & 5 & 5 & 10 & 50 \\
Total & 11 & 16 & 27 & 40,7 \\
\hline
\end{tabular}

Tabela 2. Relação entre o tempo de conhecimento do diagnóstico de ser portador de diabete e presença ou ausência de retinopatia diabética.

\begin{tabular}{lcccc}
\hline Tempo de diagnóstico & Presença & Ausência & Total & $\%$ de presença \\
\hline$<8$ anos & 2 & 10 & 12 & 16,6 \\
$8 \leq$ anos $\leq 15$ & 7 & 4 & 11 & 63,6 \\
$>15$ anos & 2 & 2 & 9 & 22,2 \\
Total & 11 & 16 & 27 & 40,7 \\
\hline
\end{tabular}

Tabela 3. Conduta do paciente no controle da glicemia.

\begin{tabular}{lcc}
\hline $\begin{array}{c}\text { Tratamento do } \\
\text { diabetes mellitus }\end{array}$ & $\begin{array}{c}\text { Frequência } \\
\text { absoluta }(\mathrm{n})\end{array}$ & $\begin{array}{c}\text { Frequência } \\
\text { relativa }(\%)\end{array}$ \\
\hline Comprimido & 18 & 58,10 \\
Comprimido e dieta & 7 & 22,60 \\
Ausente & 3 & 9,70 \\
Insulina & 2 & 6,50 \\
Dieta & 1 & 3,20 \\
\hline
\end{tabular}

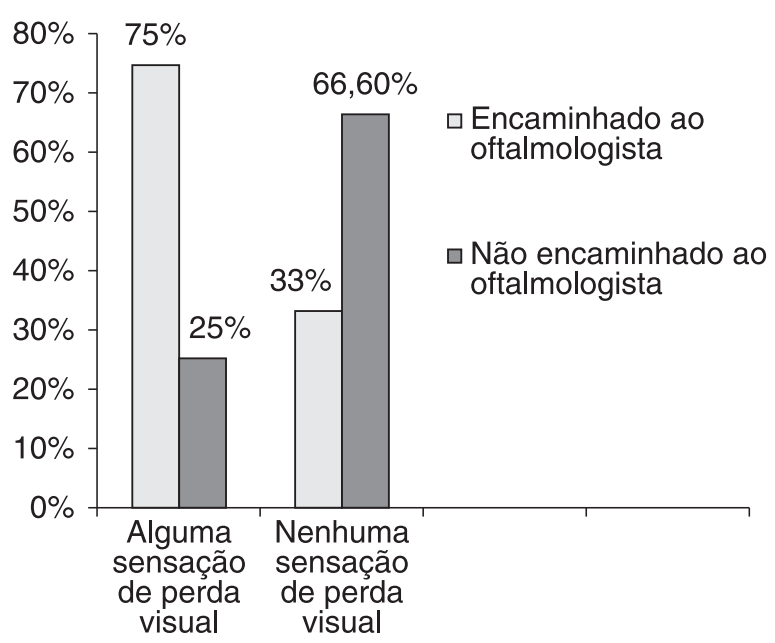

Figura 2. Opinião sobre a sensação de perda visual em relação ao encaminhamento ao oftalmologia. 


\section{Discussão}

A prevalência de RD encontrada neste estudo piloto foi de 40,7\%. Considerando o total de pacientes diagnosticados com $\mathrm{RD}$, a presença de $\mathrm{RD}$ entre os homens com diabetes foi de $45,5 \%$ e entre as mulheres foi de $54,5 \%$. Quanto ao tipo de $\mathrm{RD}$, foi diagnosticado somente R.D.N.P. Embora tenha sido encontrada uma proporção maior de RD entre as mulheres, este achado não foi estatisticamente significativo $(\mathrm{p}=0,363)$, o que condiz com os resultados obtidos na maioria da literatura, demonstrando que não há diferença de prevalência de $\mathrm{RD}$ entre os $\operatorname{sexos}^{8,10}$. Porém, contrasta com os dados achados no estudo de Andrade et al. (2006) que relevou prevalência de $\mathrm{RD}$ em homens $(56,2 \%)^{11}$.

$\mathrm{Na}$ análise da duração do diabetes, houve diferença significativa entre os grupos de presença e ausência da retinopatia, quando comparados em relação ao tempo de diagnóstico da doença. Entre todos os pacientes (11) que receberam diagnóstico presente para a RD, o tempo médio de diabete encontrado foi de 11,8 anos. Isto coincide com a literatura, que constata relação direta entre o tempo de diagnóstico da doença e a presença de retinopatia ${ }^{10}$. Chama a atenção o fato de encontrarmos, nesse grupo, dois pacientes com apenas 1 e 2 anos de tempo diagnóstico de diabetes e apresentarem retinopatia diabética.

A idade média alta encontrada do diagnóstico do DM, entre os pesquisados, é de 11,8 anos. Isso pode se dar, devido ao início insidioso da doença, pois, provavelmente, os pacientes já eram portadores de diabete melito tipo 2 bem antes do conhecimento do diagnóstico para tal doença.

Estudos estimam que, em pacientes com DM tipo 2, a doença já esteja presente há cerca de 4 a 7 anos em relação à época em que é feito o diagnóstico de DM. Esta observação explica a presença de RD já na ocasião do diagnóstico em pacientes com DM tipo $2^{12}$.

Este fato demonstra a importância do papel da ESF no controle dos fatores de risco, diminuindo assim a progressão das alteraçôes retinianas. As medidas preventivas são: controle da glicemia, da pressão arterial, a realização do diagnóstico em fase inicial e passível de intervenção.

\section{Conclusão}

Nossos achados revelaram prevalência de retinopatia diabética em 40,7\% dos pacientes portadores de DM tipo 2, cadastrados no programa HIPERDIA, da ESF do município de Ananindeua. Houve significância estatística da RD em relação ao tempo de evolução do diabetes, sendo mais prevalente $(63,6 \%)$ em pacientes com duração da doença entre 8 e 15 anos.

Estes resultados reforçam a importância da atenção primária no acompanhamento dos pacientes diabéticos, realizando orientação, prevenção e tratamento. Além disso, é fundamental a orientação aos servidores da saúde pública quanto à importância da sua atuação no controle dos fatores de risco que minimizam as lesôes causadas pelo DM.

Dessa forma, a RD deve ter uma abordagem multidisciplinar, objetivando um diagnóstico e acompanhamento em fases iniciais da doença, antes que lesóes mais graves e devastadoras da visão tenham ocorrido.

O perfil deste estudo epidemiológico contribui como fonte de dados essenciais na formulação de novas políticas públicas, visando à prevenção e diagnóstico precoce da RD na comunidade.

\section{Referências}

1. Grillo MFF, Gorini MIPC. Caracterização de pessoas com Diabetes Mellitus Tipo 2. Rev Bras Enferm. 2007; 60(1): 49-54. http://dx.doi.org/10.1590/ S0034-71672007000100009

2. Bosco A, Lerário AC, Soriano D, Santos RF, Massote P, Galvão D, et al. Retinopatia Diabética. Arq Bras Endocrinol Metab. 2005; 49(2): 60-62. PMid: 16544035 .

3. Maia Júnior OO, Marback RF, Bonanomi MTB, Takahashi WY, Kara-José N. Avaliação oftalmologia tardia em pacientes com retinopatia diabética. Rev Assoc Med Bras. 2007; 53(1): 39-43. PMid:17420892.

4. Lisboa HRK. Relação entre retinopatia diabética e dermopatia diabética em pacientesportadores de diabetes mellitus tipo 2. Rev Bras Oftalmol. 2008; 67(6): 297-300. http://dx.doi.org/10.1590/S0034-72802008000600006 
5. Boelter MC, Azevedo MJ, Gross JL, Lavinsky J. Fatores de risco para retinopatia diabética. Arq Bras Oftalmol. 2003; 66(2): 239-247. http://dx.doi. org/10.1590/S0004-27492003000200024

6. Isaac DC, Ávila MP. Condições da retinopatia diabética no Brasil. Rev SBRV. 2009; 10(2): 04-10.

7. Vasconcellos AMM. Uma análise do processo de implantação dos centros de referência para o tratamento da retinopatia diabética na Rede de Serviços do Sistema Único de Saúde do Brasil. [Dissertação]. Rio de Janeiro: Instituto de Medicina Social, Universidade do Estado do Rio de Janeiro; 2002.

8. Garcia CAA, Gomes AHB, Nunes IM, Oliveira TL, Monteiro J. Incidência e fatores de risco da retinopatia diabética em pacientes do Hospital Universitário Onofre Lopes, Natal-RN. Arq Bras Oftalmol. 2003; 66(3): 355-358. http://dx.doi.org/10.1590/S0004-27492003000300018

9. Souza AR, Moreira AL. Serviço de fisiologia. Desenvolvido pela Faculdade de Medicina da Universidade do Porto, 2006-2007. Observação do fundo ocular. [acesso em 2011 nov. 15]. Disponível em: www.fisiologia.med.up.pt

10. Santos ICRV, Carvalho EF, Souza WV, Medeiros MCWC, Nóbrega MGL, Lima PMS. Complicações crônicas dos diabéticos tipo 2 atendidos nas Unidades de Saúde da Família - PE. Arq Bras Saúde Matern Infant. 2008; 8(4): 427-433.

11. Pereira DS. Prevalência da retinopatia diabética no Ambulatório de Endocrinologia Pediátrica da Santa Casa de Misericórdia de São Paulo. Arq Bras Oftalmol. 2004; 67: 111-114. http://dx.doi.org/10.1590/S0004-27492004000100020

12. Esteves J, Laranjeira AF, Roggia MF, Dalpizol M, Scocco C, Kramer CK, et al. Fatores de risco para retinopatia diabética. Arq Bras Endocrinol Metab. 2008; 52(3): 431-441. PMid:18506268. 